\title{
A Geometrical Approach to the Boundary Element Method
}

\author{
Bernhard Auchmann ${ }^{1}$, Stefan Kurz ${ }^{2}$, and Sergej Rjasanow ${ }^{3}$ \\ ${ }^{1}$ CERN AT/MEL, H-1211 Geneva 23, Switzerland \\ ${ }^{2}$ ETAS GmbH, 70469 Stuttgart, Germany \\ ${ }^{3}$ Universität des Saarlandes, 66041 Saarbrücken, Germany
}

\begin{abstract}
We introduce a geometric formulation of the boundary element method (BEM), using concepts of the discrete electromagnetic theory. Geometric BEM is closely related to Galerkin-BEM and to the generalized collocation scheme. It is easy to implement, accurate, and computationally efficient. We validate our approach with 2-D examples and give an outlook to 3-D results.
\end{abstract}

Index Terms-Boundary element method (BEM), discrete electromagnetism.

\section{INTRODUCTION}

W E introduce a variant of the boundary element method (BEM) which is inspired by the discrete electromagnetic theory (DEM), e.g., by cell methods, finite-integration technique, and the DEM interpretation of the finite element method. We call the formulation the geometric BEM and understand that it distinguishes between topological and metric concepts. We assume that the reader is familiar with topological operators such as boundary, coboundary, trace, and pairing operators, and with the metric discrete Hodge operator of DEM [2]. For the geometric BEM we introduce an additional metric entity, the non-local, single- and double-layer double-cochains.

With this paper, we pursue a program that was started with the generalized collocation method [1]. Generalized collocation had been geared towards an introduction of geometric concepts of DEM (barycentrically dual mesh) to BEM. The collocation method was later formulated using operators of DEM such as discrete derivative- and trace operators, and it was discussed in the context of DEM-BEM coupling [2]. The geometric BEM constitutes our most consequent application of geometric concepts to BEM to date, in that it discretizes the Green integral kernel itself.

The paper is structured as follows: The representation formula and the first Calderóon equation in differential form notation is introduced in Section II. The Galerkin- and generalized-collocation methods are recalled in Section III. We then introduce the geometric BEM formulation in Section V. Information on implementation, numerical results, and validation with analytical solutions for a 2-D geometric BEM, as well as for 2-D DEM-BEM coupling are presented in Section VI. Preliminary results for the 3 -D vector-potential formulation are found in Section VII.

\section{A. Notation}

We denote $\bar{F} \in \mathcal{F}^{p}(\Omega)$ a differential $p$-form, and $\langle\bar{F} \mid m\rangle$ the integral of a differential $p$-form over a $p$-manifold $m \subset \Omega$. Stokes' theorem for differential forms reads $\langle\mathrm{d} \bar{F} \mid m\rangle=\langle\bar{F} \mid \mathrm{b} m\rangle$, with the boundary operator $\mathrm{b}$, and the coboundary or exterior derivative operator $\mathrm{d}$. We further denote $\hat{F} \in \mathcal{C}^{p}(C)$ (hat) the $p$-cochain of a field and $\{\hat{F}\} \in \mathbb{R}^{n_{p}}$

Digital Object Identifier 10.1109/TMAG.2007.916563 the coefficient vector of the cochain. $\mathcal{C}^{p}(C)$ or simply $\mathcal{C}^{p}$ denotes the space of cochains on a cell complex $C . n_{p}$ denotes the number of $p$-cells $\check{\varrho}_{p}$ in the complex $C$. The $i$ th coefficient of a cochain is obtained from a differential form by the de Rham map, $\{\hat{F}\}^{i}=\left\langle\bar{F} \mid \check{\varrho}_{p}^{i}\right\rangle$. Furthermore, $\check{c} \in \mathcal{C}_{p}(C)$ (check) denotes a chain of cells and $\{\check{c}\} \in \mathbb{R}^{n_{p}}$ the chain's coefficient vector. $\mathcal{C}_{p}(C)$ denotes the space of chains. Objects that are defined on the dual complex are underlined, e.g., $\underline{\hat{F}}$ for a dual cochain. Finally, we denote $\tilde{F} \in \mathcal{W}^{p}(C)$ (tilde) the Whitney form, that is the interpolation of a discrete field based on its coefficients $\{\hat{F}\}$. The duality product of chains and cochains is written $\langle\hat{F} \mid \check{c}\rangle=\{\hat{F}\}^{\mathrm{T}}\{\check{c}\}$, and operators acting on chains and cochains are written in bold font, e.g., $\mathbf{d}$ for the discrete derivative-, $\mathbf{b}$ for the boundary operator. In what follows, we divide the open domain $\Omega$ into open domains $\Omega^{-}, \Omega^{+}$, and $\Gamma$, with $\Omega^{+} \cap \Omega^{-}=\emptyset$ and $\Omega^{-} \cup \Omega^{+} \cup \Gamma=\Omega$.

\section{CONTINUOUS REPRESENTATION Formula}

The representation formula for a potential that is governed by the Poisson equation $\delta \mathrm{d} \bar{\alpha}=* \bar{\eta}, \bar{\alpha} \in \mathcal{F}^{p}\left(\Omega^{-}\right), \bar{\eta} \in \mathcal{F}^{n-p}\left(\Omega^{-}\right)$, $\mathrm{d} \bar{\eta}=0$ is given in [1]

$$
\bar{\alpha}^{\prime}=\underbrace{\left\langle\gamma_{\bar{D}}^{-} G^{p} \wedge \bar{\gamma} \mid \Gamma\right\rangle}_{\bar{\Psi}_{\mathrm{SL}}(\bar{\gamma})}-\underbrace{(-1)^{p}\left\langle\gamma_{\mathrm{N}} G^{p} \wedge \bar{\beta} \mid \Gamma\right\rangle}_{\bar{\Psi}_{\mathrm{DL}}(\bar{\beta})}+\left\langle G^{p} \wedge \bar{\eta} \mid \Omega^{-}\right\rangle .
$$

The representation formula is valid for dimensions $n=2,3$ and for degrees $p=0,1$ of the potential $\bar{\alpha}$. Primed objects belong to the so-called observation space and unprimed ones to the source space. ${ }^{1} G^{p}$ denotes the Green double-form

$$
\begin{aligned}
& G^{0}=g\left(x, x^{\prime}\right), \\
& G^{1}=g\left(x, x^{\prime}\right) \mathrm{d} x^{1} \otimes \mathrm{d} x^{1 \prime}+\ldots+\mathrm{d} x^{n} \otimes \mathrm{d} x^{n \prime}
\end{aligned}
$$

with the Green function $g\left(x, x^{\prime}\right)$ in 2 or 3 dimensions. We identify the single-layer, and double-layer potentials on the boundary $\Gamma$, as well as the source-term $\bar{\eta} \in \mathcal{F}^{n-p}\left(\Omega^{-}\right)$, which we will henceforth set to zero for the sake of brevity. Dirichlet and Neumann data are denoted $\bar{\beta}$ and $\bar{\gamma}$. They are obtained from the potential $\bar{\alpha}$ by action of Dirichlet and Neumann traces

$$
\begin{aligned}
& \gamma_{\mathrm{D}}^{-}: \mathcal{F}^{p}\left(\Omega^{-}\right) \rightarrow \mathcal{F}^{p}(\Gamma) \quad: \bar{\alpha} \mapsto \bar{\beta}=\mathrm{t}^{-} \bar{\alpha}, \\
& \gamma_{\mathrm{N}}^{-}: \mathcal{F}^{p}\left(\Omega^{-}\right) \rightarrow \mathcal{F}^{n-p-1}(\Gamma) \quad: \bar{\alpha} \mapsto \bar{\gamma}=\mathrm{t}^{-} * \mathrm{~d} \bar{\alpha} .
\end{aligned}
$$

${ }^{1}$ We use $\bar{\alpha}=\mathrm{id}^{*} \bar{\alpha}^{\prime}$, where id ${ }^{*}$ is the pull-back of the identity $\Omega^{\prime}=\operatorname{id}(\Omega)$. 
The following jump relations hold:

$$
\begin{aligned}
{\left[\bar{\Psi}_{\mathrm{SL}}(\bar{\gamma})\right]_{\Gamma} } & =0, \\
{\left[\bar{\Psi}_{\mathrm{DL}}(\bar{\beta})\right]_{\Gamma} } & =\bar{\beta}^{\prime}
\end{aligned}
$$

where the jump of a form $\bar{F}$ is defined as $[\bar{F}]_{\Gamma}=\left(\gamma_{\mathrm{D}}^{+\prime} \bar{F}-\right.$ $\left.\gamma_{\mathrm{D}}^{-\prime} \bar{F}\right)$. Applying $\gamma_{\mathrm{D}}^{-\prime}$ to the representation formula, we obtain the first Calderón equation

$$
\bar{\beta}^{\prime}=\gamma_{\mathrm{D}}^{-\prime} \bar{\Psi}_{\mathrm{SL}}(\bar{\gamma})-\gamma_{\mathrm{D}}^{-\prime} \bar{\Psi}_{\mathrm{DL}}(\bar{\beta}) .
$$

With $\bar{\Psi}_{\widetilde{\mathrm{DL}}}=\Theta_{\mathrm{r}}\left(\gamma_{\mathrm{D}}^{-\prime} \bar{\Psi}_{\mathrm{DL}}+\gamma_{\mathrm{D}}^{+\prime} \bar{\Psi}_{\mathrm{DL}}\right)$ and the above jump relations, the equation reads ${ }^{2}$

$$
\Theta_{\mathrm{r}} \bar{\beta}^{\prime}=\gamma_{\mathrm{D}}^{-} \bar{\Psi}_{\mathrm{SL}}(\bar{\gamma})-\bar{\Psi}_{\widetilde{\mathrm{DL}}}(\bar{\beta})
$$

where $\Theta_{\mathrm{r}}:=\Theta /(n-1) 2 \pi$ denotes the relative solid angle under which the domain is seen from the observation point. On smooth domain boundaries $\Theta_{\mathrm{r}}$ equals $1 / 2$.

\section{Galerkin AND Generalized Collocation Methods}

For the sake of brevity we only discuss the definition of the single-layer matrix in a Dirichlet problem. The Neumann data is approximated in the space of Whitney $q$-forms, $q=n-p-1$, on the discrete boundary $\Gamma_{h} . \tilde{w}_{i}^{q} \in \mathcal{W}^{q}\left(\Gamma_{h}\right)$ denotes the $i$ th basis Whitney $q$-form in $\Gamma_{h}$.

An element in the single-layer matrix $[\mathrm{G}]$ is defined in the Galerkin approach as

$$
\left[\mathrm{G}_{\text {Galerkin }}\right]^{i j}=\left\langle\gamma_{\mathrm{D}}^{-\prime}\left\langle\gamma_{\mathrm{D}}^{-} G^{p} \wedge \tilde{w}_{j}^{q} \mid \Gamma_{h}\right\rangle \wedge \tilde{w}_{i}^{q} \mid \Gamma_{h}^{\prime}\right\rangle
$$

whereas the generalized-collocation approach simply integrates the first Calderón equation along dual $p$-cells $\underline{\underline{e}}_{p}^{i}$

$$
\left[\mathrm{G}_{\text {colloc. }}\right]^{i j}=\left\langle\gamma_{\mathrm{D}}^{-\prime}\left\langle\gamma_{\mathrm{D}}^{-} G^{p} \wedge \tilde{w}_{j}^{q} \mid \Gamma_{h}\right\rangle \mid \underline{\varrho}_{p}^{i \prime}\right\rangle .
$$

For $n=3, p=1$, i.e., a vector-potential formulation of a magnetostatic problem on the boundary of a 3-D domain, we observe that the Galerkin method needs to perform double surface integrals for each matrix entry, whereas the collocation method integrates over the surface and the dual edge. The collocation method, however, leads to an unsymmetric single-layer matrix, whereas the Galerkin single-layer matrix is symmetric.

\section{Discrete Wedge Product ANd Pairing Matrix}

We propose to replace the Green double-form in the Galerkin approach of (2) by a double-cochain, and to use the discrete wedge product rather than the continuous one.

We therefore introduce the discrete wedge product of cochains $\wedge: \mathcal{C}^{p} \times \mathcal{C}^{q} \rightarrow \mathcal{C}^{p+q}$, that is defined using Whitney and de Rham maps (interpolation $\boldsymbol{\omega}$ and discretization $\pi$ ) [3]

$$
\hat{G} \wedge \hat{F}=\pi(\boldsymbol{\omega} \hat{G} \wedge \boldsymbol{\omega} \hat{F}) .
$$

$2 \bar{\Psi}_{\widetilde{\mathrm{DI}}}$ represents the Cauchy principal value of the singular double-layer integral in the first Calderón equation. The principal value can be numerically or analytically evaluated.
In particular for $\hat{G} \in \mathcal{C}^{p}$ and $\hat{F} \in \mathcal{C}^{n-p}$ we find

$$
\begin{aligned}
\langle\hat{G} \wedge \hat{F} \mid C\rangle & =\langle\boldsymbol{\omega} \hat{G} \wedge \boldsymbol{\omega} \hat{F} \mid C\rangle \\
& =\{\hat{F}\}^{\mathrm{T}}\left[\mathrm{P}^{p}\right]\{\hat{G}\} .
\end{aligned}
$$

The matrix $\left[\mathrm{P}^{p}\right]$ is called the pairing matrix. The element matrix $\left[\mathrm{P}_{\mathrm{e}}^{p}\right]$ is defined for a canonically oriented cell by

$$
\left[\mathrm{P}_{\mathrm{e}}^{p}\right]^{i j}=\left\langle\tilde{w}_{j}^{p} \wedge \tilde{w}_{i}^{n-p} \mid C\right\rangle .
$$

An alternative interpretation of the pairing matrix is outlined in [4]: The pairing matrix can be read as a mapping from cochains on the primal complex into cochains on the barycentrically dual cell complex, $\{\underline{\hat{E}}\}=\left[\mathrm{P}^{p}\right]\{\hat{F}\}$. We will refer to this remark later on.

\section{GEOMETRIC BEM}

\section{A. Discrete First Calderón Equation}

A discrete version of the first Calderón equation uses double cochains, or cochain-valued cochains, $\hat{\Psi}_{\mathrm{SL}} \in \mathcal{C}^{p}\left(\Gamma_{h}\right) \otimes \mathcal{C}^{p}\left(\Gamma_{h}^{\prime}\right)$ and $\hat{\Psi}_{\widetilde{\mathrm{DL}}} \in \mathcal{C}^{n-p-1}\left(\Gamma_{h}\right) \otimes \mathcal{C}^{p}\left(\Gamma_{h}^{\prime}\right)$ and reads

$$
\left\langle\hat{\Psi}_{\mathrm{SL}} \wedge \hat{\gamma} \mid \Gamma_{h}\right\rangle-\left(\iota_{\Theta} \hat{\beta}+\left\langle\hat{\Psi}_{\widetilde{\mathrm{DL}}} \wedge \hat{\beta} \mid \Gamma_{h}\right\rangle\right)=0
$$

where we use the operator $\iota_{\Theta}: \mathcal{C}^{p}\left(\Gamma_{h}\right) \rightarrow \mathcal{C}^{p}\left(\Gamma_{h}^{\prime}\right)$ that is the identity operator times the solid angle factor in (1). Note that to each $p$-cell in $\Gamma_{h}$ corresponds one solid angle.

Equation (4) is a cochain-valued equation. We interpolate the cochain by Whitney forms, and solve the Neumann problem with the Galerkin method. The resulting system of equations reads in matrix notation

$$
\begin{aligned}
{\left[\mathrm{P}^{p}\right]\left[\hat{\Psi}_{\mathrm{SL}}^{p}\right]\left[\mathrm{P}^{p}\right]^{\mathrm{T}} } & \{\hat{\gamma}\} \\
& -\left[\mathrm{P}^{p}\right]\left(\left[\Theta_{\mathrm{r}}\right]+\left[\hat{\Psi}_{\widetilde{\mathrm{DL}}}^{p}\right]\left[\mathrm{P}^{n-p-1}\right]^{\mathrm{T}}\right)\{\hat{\beta}\}=0
\end{aligned}
$$

where the single-layer matrix is defined by a double de Rham-map of the Green double-form $G^{p}$

$$
\left[\hat{\Psi}_{\mathrm{SL}}^{p}\right]^{i j}=\left\langle\gamma_{\mathrm{D}}^{-\prime}\left\langle\gamma_{\mathrm{D}}^{-} G^{p} \mid \check{\varrho}_{p}^{j}\right\rangle \mid \varrho_{p}^{i \prime}\right\rangle \text { for } i \neq j .
$$

The case $i=j$ needs to be considered separately for every pair of degree $p$ and dimension $n$, see Section VI. The discrete double-layer potential is defined accordingly

$$
\left[\hat{\Psi}_{\widetilde{\mathrm{DL}}}^{p}\right]^{i j}=\left\langle\gamma_{\mathrm{D}}^{-\prime}\left\langle\gamma_{\mathrm{N}}^{-} G^{p} \mid \check{\varrho}_{n-p-1}^{j}\right\rangle \mid \varrho_{p}^{i \prime}\right\rangle .
$$

$\left[\Theta_{\mathrm{r}}\right]$ denotes the diagonal matrix of solid angles.

The only difference between (5) and a conventional Galerkin-BEM lies in the discretization of the Green double-forms by double-cochains in (6) and (7), and their subsequent interpolation by Whitney-forms in the observationand source spaces.

The coefficients of the double cochains $\hat{\Psi}_{\mathrm{SL}}$ and $\hat{\Psi}_{\widetilde{\mathrm{DL}}}$ are metric dependent and non-local, i.e., they give rise to fully populated matrix representatives. The single-layer matrix is symmetric.

\section{B. Impact on BEM Implementation}

The pairing matrix $\left[\mathrm{P}^{p}\right]$ is topological, i.e., its element matrix only depends on the element type and connectivity, and the ma- 

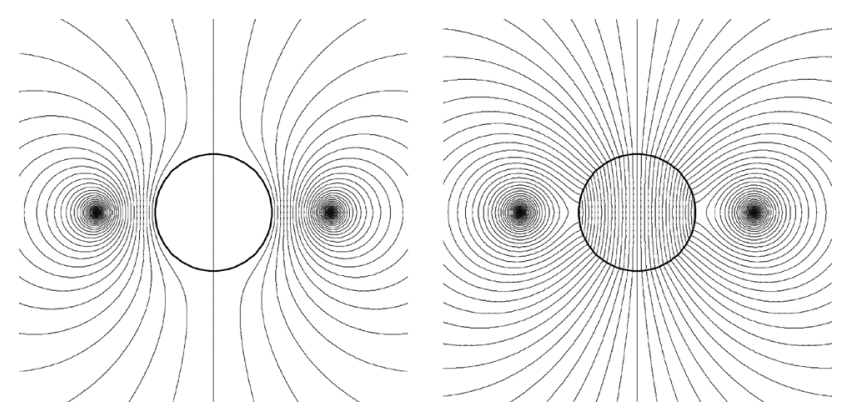

Fig. 1. Analytical solution for a cylinder in the field of a current loop. Left: With homogeneous Dirichlet boundary conditions on the cylinder surface; Right: With homogeneous Neumann boundary conditions.

trix is assembled, rather than computed. The only integration in the setup of the geometrical BEM matrices therefore happens in the de Rham maps of (6) and (7). Note that for $n=3, p=1$, the vector-potential formulation of magnetostatics, the integration domains are the edges on the primal mesh. No integration over faces is required! For $p=0$ the computation of the single-layer matrix consists of $n_{0} \times n_{0}$ evaluations of the Green function.

\section{Structural Similarities of Geometrical, Galerkin, and Collocation BEM}

We recall that the pairing matrix can be interpreted with (3) as the agent of the discrete wedge product acting between two cochains. This interpretation connects geometric BEM and Galerkin BEM. Unlike the Galerkin method, the integral kernels of single- and double-layer potentials are discretized by de Rham maps and subsequently interpolated by Whitney forms. ${ }^{3}$

If we introduce a barycentrically dual mesh, we may read the left pairing matrices in (5) as mappings of cochains on the primal mesh into cochains on the barycentrically dual mesh. Comparison with the generalized collocation method for BEM reveils the similarities between geometric-, collocation-, and Galerkin-type BEM formulations. While the collocation method discretizes the integral kernel in the observation space on the topologically dual mesh, the geometric BEM discretizes it on the primal mesh and maps it to the dual mesh by the action of the pairing matrix. This reasoning explains, why single-layer matrices from a collocation method are observed to be almost symmetric. Geometric BEM yields symmetric single-layer matrices.

\section{NumERICAL RESUlTS IN TwO DimENSIONS}

All results displayed in this and the following section were obtained with MathWorks MATLAB 7.4.

\section{A. Homogeneous Dirichlet and Neumann Problems}

In this section, we solve the homogeneous Dirichlet and Neumann problems in 2-D for a cylinder in the field of a current loop. Analytical solutions, as depicted in Fig. 1, are obtained by use of the method of image currents, compare Fig. 2 (right).

\footnotetext{
${ }^{3}$ This step compares to a similar approach in the interpretation of Galerkin FEM as DEM, where the field on the FEM right-hand side is first discretized by a de Rham map, and then interpolated by Whitney forms [5]. The pairing matrix in the right-hand side of the DEM (11) follows from this procedure.
}
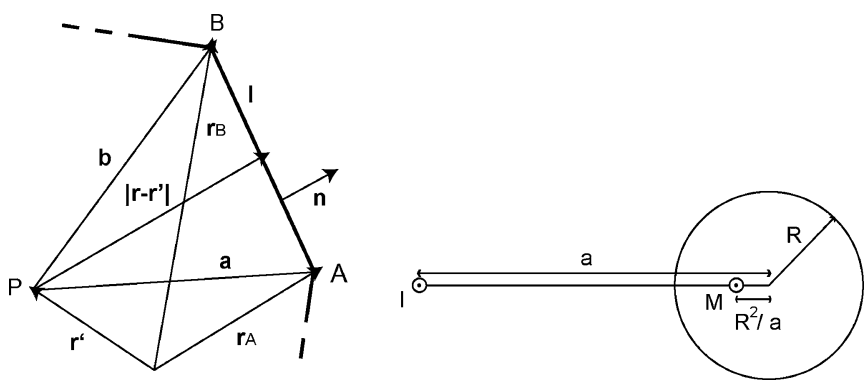

Fig. 2. Left: Notation in (8). Right: Position of a line current $I$ and a mirror current $M$ with respect to a cylinder.

For the homogeneous Neumann problem, we solve

$$
\left[\mathrm{P}^{0}\right]\left(\left[\Theta_{\mathrm{r}}\right]+\left[\hat{\Psi}_{\mathrm{DL}}^{0}\right]\left[\mathrm{P}^{1}\right]^{\mathrm{T}}\right)\left\{\hat{A}_{z}\right\}=\left[\mathrm{P}^{0}\right]\left\{\hat{A}_{z, \mathrm{~s}}\right\}
$$

where the $z$-component of the magnetic vector potential is the unknown 0-cochain and the source potential on the right-hand side is calculated from the two line currents using Biot-Savart law. The double-layer matrix is calculated from [6]

$$
\begin{aligned}
& {\left[\hat{\Psi}_{\mathrm{DL}}^{0}\right]^{i j}=\left\langle\gamma_{\mathrm{D}}^{-\prime} \gamma_{\mathrm{N}}^{-} \frac{1}{2 \pi} \ln \left(R_{0} /\left|\mathbf{r}^{i \prime}-\mathbf{r}\right|\right) \mid \check{\varrho}_{1}^{j}\right\rangle} \\
& =-\frac{\mathbf{n} \cdot \mathbf{a}}{2 \pi} \begin{cases}0, & \mathbf{a} \cdot \mathbf{b}=0 \\
\frac{|\mathbf{l}|}{|\mathbf{a} \times \mathbf{b}|}\left(\operatorname{atan} \frac{|\mathbf{a} \times \mathbf{b}|}{\mathbf{a} \cdot \mathbf{b}}+\frac{\pi}{2}(1-\operatorname{sgn}(\mathbf{a} \cdot \mathbf{b}))\right), & \mathbf{a} \cdot \mathbf{b} \neq 0 \\
\frac{\mid \mathbf{l}}{|\mathbf{a}||\mathbf{b}|}, & \mathbf{a} \cdot \mathbf{b}=0 .\end{cases}
\end{aligned}
$$

The vectors $\mathbf{a}, \mathbf{b}, \mathbf{n}$, and 1 are defined in Fig. 2 (left). $R_{0}$ is an arbitrary reference radius. The element matrix of the pairing matrix is simply given by

$$
\left[\mathrm{P}_{\mathrm{e}}^{0}\right]=\left(\begin{array}{ll}
\frac{1}{2} & \frac{1}{2}
\end{array}\right), \quad\left[\mathrm{P}^{1}\right]=\left[\mathrm{P}^{0}\right]^{\mathrm{T}} .
$$

The error of the BEM solution with respect to the analytic solution is displayed in Fig. 3 (left) as a function of mesh size. It is measured in the relative $L_{2}$-norm

$$
\delta(h)=\frac{\left\|\bar{A}_{z}-\bar{A}_{z, \text { ana }}\right\|_{L_{2}\left(\Gamma_{h}\right)}}{\left\|\bar{A}_{z, \text { ana }}\right\|_{L_{2}\left(\Gamma_{h}\right)}} .
$$

We observe convergence of the order $O\left(h^{3}\right)$ for the homogeneous Neumann problem.

The homogeneous Dirichlet problem, Fig. 1 (right), reads

$$
-\left[\mathrm{P}^{0}\right]\left[\hat{\Psi}_{\mathrm{SL}}^{0}\right]\left[\mathrm{P}^{0}\right]^{\mathrm{T}}\{\hat{H}\}=\left[\mathrm{P}^{0}\right]\left\{\hat{A}_{z, \mathrm{~s}}\right\} .
$$

The single-layer matrix is defined as

$$
\left[\hat{\Psi}_{\mathrm{SL}}^{0}\right]^{i j}= \begin{cases}\frac{\mu}{2 \pi} \ln \left(R_{0} /\left|\mathbf{r}^{i \prime}-\mathbf{r}^{j}\right|\right), & \text { for } i \neq j \\ \frac{\mu}{2 \pi} \ln \left(R_{0} / \varepsilon\right), & \text { for } i=j\end{cases}
$$

where we use the regularization constant $\epsilon$ for the otherwise singular main-diagonal entries of $\left[\hat{\Psi}_{\mathrm{SL}}^{0}\right]$. To determine the optimal regularization constant, we proceeded in a heuristic way. We used an optimization routine to minimize the relative error with respect to the analytical solution. It turns out, and we state without proof nor explanation, that an optimal regularization 

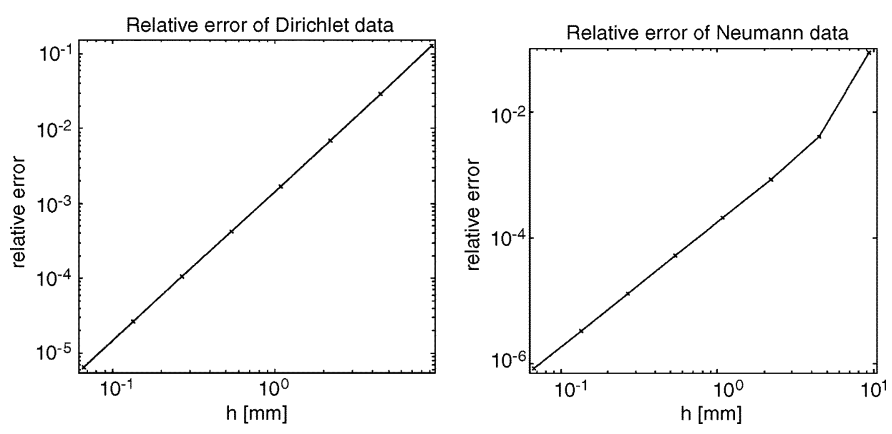

Fig. 3. Relative error of the solution of the homogeneous Neumann (left) and Dirichlet (right) problems as a function of the mesh size $h$. Note that these high rates of convergence might be examples of superconvergence where lower order error terms cancel out due to the high symmetry of the model problem.
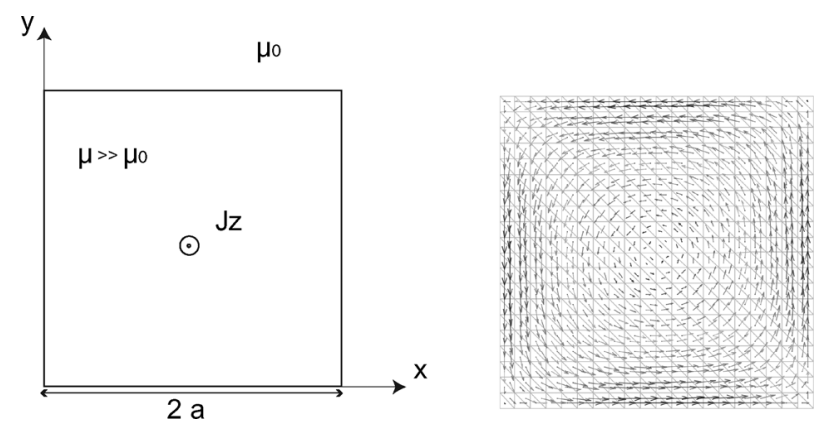

Fig. 4. Current-carrying square rod of side length $2 a=10 \mathrm{~mm}$ with magnetic permeability $\mu \gg \mu_{0}$. The field is concentrated inside the rod.

constant is given by $\epsilon=h / 2 \pi$. The geometric BEM formulation of the homogeneous Dirichlet problem converges to the analytic solution with order $O\left(h^{2}\right)$, see Fig. 3 (right).

At this point, we must mention that the pairing matrix $\left[\mathrm{P}^{0}\right]$ is singular with kernel dimension 1 for an even number of nodes in the boundary, and regular for an odd number of nodes. The kernel basis vector is the alternating vector, in which neighbouring nodes are assigned +1 and -1 , respectively. To date, we have no physical explanation for this kernel. Algebraic regularization needs to be foreseen for the equation system to be solvable.

\section{B. DEM-BEM Coupling}

We solve the system of equations

$$
\begin{array}{r}
{\left[\underline{\mathrm{D}}^{1}\right]\left[\mathrm{M}_{\nu}^{1}\right]\left[\mathrm{D}^{0}\right]\left\{\hat{A}_{z}\right\}+\left[\underline{\mathrm{J}}^{1}\right]\left[\mathrm{P}_{\mathrm{b}}^{1}\right]\left\{\hat{H}_{\mathrm{b}}\right\}=\left[\mathrm{P}^{2}\right]\left\{\hat{j}_{\mathrm{s}}\right\}} \\
{\left[\mathrm{P}^{p}\right]\left(\left[\hat{\Psi}_{\widetilde{\mathrm{DL}}}^{p}\right]\left[\mathrm{P}^{n-p-1}\right]^{\mathrm{T}}+\left[\Theta_{\mathrm{r}}\right]\right)\left[\mathrm{T}^{1}\right]\left\{\hat{A}_{z}\right\}-} \\
{\left[\mathrm{P}^{p}\right]\left[\hat{\Psi}_{\mathrm{SL}}^{p}\right]\left[\mathrm{P}^{p}\right]\left\{\hat{H}_{\mathrm{b}}\right\}=0}
\end{array}
$$

i.e., the discrete curl-curl equation including the boundary term, and the geometric BEM. $\hat{H}_{\mathrm{b}}$ denotes the magnetic-field cochain on the boundary complex. The discrete Hodge operator $\left[\mathrm{M}_{\nu}^{1}\right]$ with the inverse magnetic permeability $\nu$ may be the Galerkin Hodge operator [5], or, as in our case, the geometric discrete Hodge operator of [7]. [ $\left.\underline{J}^{1}\right]$ is the jump or coclosure operator [4], and $\left[\mathrm{T}^{1}\right]$ denotes the discrete trace operator, $\left[\underline{\mathrm{J}}^{p}\right]=\left[\mathrm{T}^{n-p-1}\right]^{\mathrm{T}}$.

We solve the model problem of a highly permeable, currentcarrying rod of side length $10 \mathrm{~mm}$ in Fig. 4. An analytical solution of the problem is given in [8]. We compare the results in a relative energy norm and find convergence of order $O\left(h^{2}\right)$, e.g.,
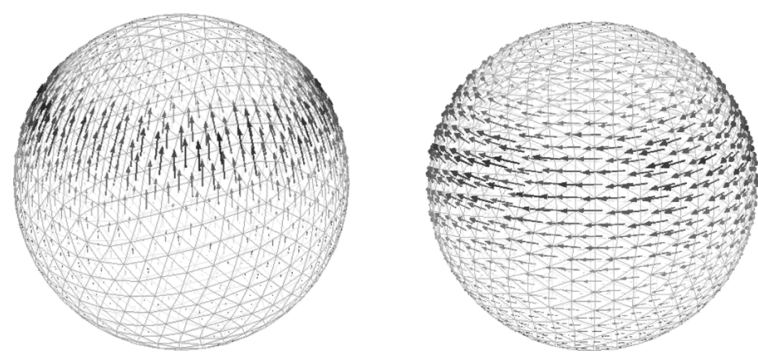

Fig. 5. Solutions of the homogeneous Dirichlet- and Neumann problems of a sphere in the field of a loop-current that is placed around the upper hemisphere.

for mesh sizes of 2,1 , and $0.5 \mathrm{~mm}$ we obtain relative errors of 18.9, 4.7, and 1.2 units $10^{-3}$, respectively.

\section{PReliminary Results in Three Dimensions}

We have implemented single- and double-layer potentials in 3 -D for $p=1$, i.e., the vector-potential formulation of magnetostatics. The homogeneous Dirichlet and Neumann problems on a sphere have been solved . First results indicate linear convergence for the homogeneous Dirichlet problem. The discussion of algebraic properties, and of convergence in appropriate error norms will be published in the near future. Fig. 5 displays first results.

\section{CONCLUSION}

We have introduced a geometric formulation of BEM, which represents a Galerkin-type BEM with discrete integral kernels. The involved matrices are easily implemented. Whitney elements need not be implemented at all for the presented variants of BEM and of DEM-BEM coupling. The method is found to be accurate and computationally efficient for $n=2, p=0$. The case $n=3, p=1$ is under investigation and $n=3, p=0$ will follow shortly. Like Galerkin and unlike collocation BEM, geometric BEM yields symmetric single-layer matrices. The interpretation of pairing matrices in geometric BEM unveils the conceptual proximity of Galerkin and collocation BEM.

\section{REFERENCES}

[1] S. Kurz, O. Rain, V. Rischmueller, and S. Rjasanow, "Discretization of boundary integral equations by differential forms on dual grids," IEEE Trans. Magn., vol. 40, no. 2, pp. 826-829, Mar. 2004.

[2] B. Auchmann, S. Kurz, O. Rain, and S. Russenschuck, "Algebraic properties of BEM-FEM coupling with Whitney elements," COMPEL, vol. 24, no. 2, pp. 480-494, Feb. 2005.

[3] S. O. Wilson, "On the algebra and geometry of a manifold's chains and cochains," Ph.D. thesis, Stony Brook Univ., Stony Brook, NY, Aug. 2005.

[4] B. Auchmann and S. Kurz, "The pairing matrix in discrete electromagnetism: On the geometry of discrete de Rham currents," Eur. Phys. J.-Appl. Phys., 2007, accepted for publication.

[5] T. Tarhasaari, L. Kettunen, and A. Bossavit, "Some realizations of a discrete Hodge operator: A reinterpretation of finite element techniques," IEEE Trans. Magn., vol. 35, no. 3, pp. 1494-1497, May 1999.

[6] H. Haas, Numerische Berechnung Elektromagnetischer Felder. Lecture notes, Vienna Univ. Technol., 1999.

[7] B. Auchmann and S. Kurz, "A geometrically defined discrete Hodge operator on simplicial cells," IEEE Trans. Magn., vol. 42, no. 4, pp. 643-646, Apr. 2006

[8] N. Schwerg, "Vorstellung unterschiedlicher Lösungsverfahren zur Berechnung der Induktivität eines hochpermeablen zylindrischen Leiters-Anwendung der Variationsrechnung," TET Note 2007-5-1, Theoretische Elektrotechnik TU Berlin, May 2007.

Manuscript received June 24, 2007. Corresponding author: B. Auchmann (e-mail: bernhard.auchmann@cern.ch). 